\title{
Utilisation du lupin (Lupinus albus L.) chez le poulet de chair pendant la période de finition
}

\author{
G. UZU \\ Developpement Alimentation animale \\ A.E.C., F 03600 Commentry
}

\section{Résumé}

L'utilisation du lupin blanc doux (Lupinus albus L.) chez le poulet de chair âgé de 4 à 7 semaines est étudiée dans deux expériences. Dans la première, tous les régimes ont des teneurs en protéines élevées $(19$ p. 100). Le régime témoin est de type pratique maïs-soja, supplémenté ou non en arginine. Les autres régimes renferment du lupin (40 p. 100) en remplacement du tourteau de soja, et sont équilibrés en acides aminés essentiels par des supplémentations en L-Lysine $\mathrm{HCl}$, DL-Méthionine, et L-Tryptophane. Dans la deuxième expérience, nous étudions l'influence d'une réduction du taux protidique dans les régimes maïs-lupin-farine de viande, supplémentés ou non avec des acides aminés de synthèse.

Le remplacement du tourteau de soja par du lupin blanc doux n'affecte pas les performances lorsque le régime est rééquilibré en acides aminés essentiels (lysine, méthionine et tryptophane). L'apport important d'arginine lié à l'introduction du lupin ne semble donc pas préjudiciable. D'ailleurs, l'addition d'arginine de synthèse dans un régime maïstourteau de soja, permettant d'obtenir une teneur en arginine identique à celle du régime maïs-lupin (1,66 p. 100), apparaît sans effet sur les performances.

La réduction du taux protidique de 20 à 17 p. 100, par une diminution de la teneur en lupin du régime, se traduit par une baisse des performances de croissance. Cependant, l'addition simultanée de lysine, de méthionine et de tryptophane permet de rétablir les performances zootechniques à un niveau équivalent à celui des lots témoins.

\section{Introduction}

La possibilité d'utilisation du lupin dans l'alimentation animale est liée à la sélection de lignées dépourvues (ou très pauvres) en alcaloïdes. Actuellement, trois espèces de lupin «doux» présentent un intérêt agronomique : le lupin bleu ( $L$. angustifolius), le lupin jaune (L. luteus) et le lupin blanc (L. albus).

Le lupin bleu, associé à des farines de viande, est largement utilisé en Australie. Les performances de croissance du poulet obtenues avec de tels régimes sont tout à fait comparables à celles réalisées avec des aliments de type céréales - tourteau de soja (Gladstones, 1977). 
Le lupin jaune peut remplacer partiellement le tourteau de soja dans l'aliment poulet en croissance. Selon VogT, Harnish \& Krieg (1979), le taux d'incorporation ne doit pas cependant dépasser $10,20,30$ et 40 p. 100 respectivement pendant la première, la deuxième, la troisième et la quatrième semaine d'âge.

Dans le cas du lupin blanc, son introduction à raison de 40 p. 100 dans le régime semble préjudiciable aux performances des poulets pendant la période de finition (LACASSAGNe, 1979). Cette faible efficacité pourrait être liée à la composition en acides aminés de cette matière première. Comparée au tourteau de soja, la graine de lupin se caractérise par une teneur très élevée en arginine et par une déficience relative en lysine, méthionine et tryptophane.

Pour vérifier cette hypothèse, nous étudions dans un premier essai l'influence d'un apport excessif d'arginine dans un aliment maïs - tourteau de soja, ainsi que les effets de différentes supplémentations en lysine et en méthionine dans un régime maïs-lupin rééquilibré en tryptophane.

Dans le deuxième essai, nous utilisons des régimes renfermant du maïs, du lupin et de la farine de viande, et nous recherchons l'effet d'une réduction du taux protidique en ajoutant ou non des acides aminés essentiels (L-lysine HCl, DL-méthionine, L-thréonine et L-tryptophane).

\section{Matériel et méthodes}

Dans les deux expériences, les poulets de chair mâles sont issus d'un croisement commercial (ISA-JV 715). Pendant les quatre premières semaines, ils reçoivent à volonté un aliment maïs - tourteau de soja-suif, contenant $3200 \mathrm{kcal}$ d'énergie métabolisable et 21,5 p. 100 de protéines.

De 4 à 7 semaines, ils sont répartis en lots selon la méthode des blocs complets équilibrés, et reçoivent les aliments expérimentaux. Les caractéristiques analytiques des graines de lupin utilisées sont présentées dans le tableau 1.

Dans la première expérience, on répartit 660 poulets en 11 lots de 60 animaux chacun (5 par cage). Les 11 régimes utilisés sont isoénergétiques (3130 kcal d'énergie métabolisable) et isoprotidiques (tabl. 2). Les deux premiers sont à base de maïs et de tourteau de soja, supplémentés ou non avec 0,5 p. 100 d'arginine. Cette supplémentation permet d'atteindre le niveau du régime à base maïs-lupin. Les 9 autres régimes maïs-lupin sont supplémentés selon un plan factoriel avec de la L-Lysine $\mathrm{HCl}$ $(0,08,0,18$ et 0,26 p. 100 du régime) et de la DL-Méthionine $(0,11,0,24$ et 0,37 p. 100 du régime).

Dans la deuxième expérience, à partir de trois régimes de base maïs-lupin isoénergétiques (3 $200 \mathrm{kcal}$ d'énergie métabolisable $/ \mathrm{kg}$ ), mais de teneurs en protéines différentes $(20,18,5$ et 17 p. 100), on constitue 16 régimes en faisant varier les apports de lysine, méthionine, thréonine et tryptophane. Les niveaux de supplémentations de ces acides aminés varient en fonction du taux protidique, et sont conformes aux recommandations de l'A.E.C. (1978). Chaque lot contient 60 poulets. 


\section{Tableau 1}

Caractéristiques analytiques des graines de lupin utilisées.

Analytical characteristics of lupin grains utilized.

\begin{tabular}{|c|c|c|}
\hline \multirow{2}{*}{ Résultats d'analyses - Analytical results } & \multicolumn{2}{|c|}{ Expériences - Experiments } \\
\hline & 1 & 2 \\
\hline Matière sèche - Dry matter (p. 100) ... & 86,2 & 85,0 \\
\hline $\begin{array}{c}\text { Protéines brutes - Crude protein (p. 100) } \cdots \cdots \cdots \cdots \cdots \\
\text { P. } 100 \text { M.A.T. - C.P. }\end{array}$ & 33,6 & 31,2 \\
\hline Lysine & 4,70 & 5,10 \\
\hline Méthionine $\ldots$. & 0,60 & 0,75 \\
\hline Méthionine + cystine & 1,95 & 2,10 \\
\hline Thréonine & 3,60 & 3,60 \\
\hline Arginine ... & 10,20 & 11,20 \\
\hline Glycine $\ldots .$. & 3,80 & 3,90 \\
\hline Glycine + sérine & 8,60 & 9,00 \\
\hline 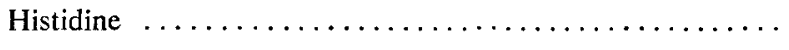 & 2,10 & 1,90 \\
\hline Isoleucine $\ldots .$. & 4,30 & 4,60 \\
\hline 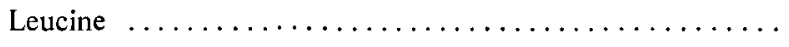 & 7,10 & 7,20 \\
\hline Phénylalanine $\ldots \ldots \ldots \ldots \ldots$ & 3,70 & 3,80 \\
\hline Phénylalanine + tyrosine & 8,10 & 8,20 \\
\hline Valine & 4,20 & 4,30 \\
\hline \multicolumn{3}{|l|}{ Valeurs estimées - Estimated values } \\
\hline Energie métabolisable - Metabolizable energy (kcal/kg) & \multicolumn{2}{|c|}{2480} \\
\hline Tryptophane - Tryptophan (p. 100 M.A.T. - C.P.) & \multicolumn{2}{|c|}{0,69} \\
\hline Cellulose brute - Crude fiber (p. 100) & \multicolumn{2}{|c|}{10} \\
\hline
\end{tabular}




\section{TABleau 2}

Composition et caractéristiques des régimes de base (expérience 1).

Composition and characteristics of basal diet (experiment 1).

\begin{tabular}{|c|c|c|}
\hline Matières premières - Ingredients (p. 100) & $\begin{array}{c}\text { Avec tourteau soja } \\
\text { With soyabean } \\
\text { neal }\end{array}$ & $\begin{array}{l}\text { Avec lupin } \\
\text { With lupin }\end{array}$ \\
\hline Maïs - Maize $\ldots \ldots \ldots \ldots \ldots \ldots \ldots \ldots \ldots \ldots \ldots \ldots \ldots \ldots$ & 66,33 & 47,21 \\
\hline Tourteau de soja - Soybean meal (50 p. 100) ..... & 24,5 & - \\
\hline 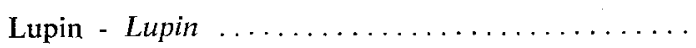 & - & 40 \\
\hline Gluten de maïs $60-$ Maize gluten $\ldots \ldots \ldots \ldots$ & 2 & 2 \\
\hline Huile de maïs - Maize oil . ............. & - & 3,5 \\
\hline Suif - Tallow $\ldots \ldots \ldots \ldots \ldots \ldots \ldots \ldots \ldots \ldots \ldots \ldots$ & 3 & 3 \\
\hline 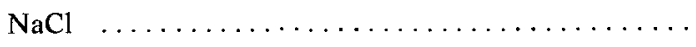 & 0,4 & 0,4 \\
\hline Carbonate de $\mathrm{Ca}-\mathrm{Ca}$ carbonate $\ldots . . \ldots \ldots \ldots$ & 1,4 & 1,4 \\
\hline Phosphate bicalcique - Dicalcium phosphate ..... & 1,925 & 1,925 \\
\hline Oligo éléments - Trace elements $\left(^{*}\right) \ldots \ldots \ldots$ & 0,05 & 0,05 \\
\hline Vitamines - Vilamins $\left(^{* *}\right) \ldots \ldots \ldots \ldots$ & 0,225 & 0,225 \\
\hline $\begin{array}{l}\text { D.L. Méthionine } \ldots \ldots \ldots \ldots \ldots \ldots \ldots \ldots \ldots \\
\text { L. Lysine } \mathrm{HCl} \ldots \ldots \ldots \ldots \ldots \ldots \ldots \ldots \\
\text { L. Tryptophane } \ldots \ldots \ldots \ldots \ldots \ldots \ldots\end{array}$ & $\begin{array}{l}0,13 \\
0,04 \\
-\end{array}$ & $\begin{array}{l}0,11 \\
0,10 \\
0,08\end{array}$ \\
\hline Caractéristiques - Characteristics & & \\
\hline $\begin{array}{l}\text { Energie métabolisable - Metabolizable energy } \\
(\mathrm{kcal} / \mathrm{kg}) \ldots \ldots \ldots \ldots \ldots \ldots \ldots \ldots \ldots \ldots \ldots \ldots \ldots\end{array}$ & 3130 & 3130 \\
\hline Protéines brutes - Crude protein $(\mathrm{N} \times 6,25)$. & 19,1 & 18,8 \\
\hline Lysine (p. 100) $\ldots \ldots \ldots \ldots \ldots \ldots \ldots$ & 0,96 & 0,77 \\
\hline Méthionine (p. 100) ........... & 0,44 & 0,22 \\
\hline Méthionine + Cystine (p. 100) & 0,77 & 0,53 \\
\hline Arginine $(p .100) \ldots \ldots \ldots \ldots \ldots \ldots \ldots \ldots \ldots$ & 1,15 & 1,66 \\
\hline Tryptophane - Tryptophan (p. 100) ............ & 0,22 & 0,22 \\
\hline
\end{tabular}

(*) En p.p.m. : Sulfate de cobalt : 1,$2 ;$ Sulfate de cuivre : 32 ; Sulfate de fer : 160 ; Iodate de calcium : 1,25; Oxyde de manganèse : 156 ; Sélénite de soude : 0,35; Oxyde de zinc : 50 .

(**) Pour $100 \mathrm{~kg}$ d'aliment : Vitamine A : 1500000 U.I.; Vitamine D3 : 300000 U.I. ; Vitamine E : $3 \mathrm{~g}$; Vitamine B1:0,2 g; Vitamine B2:2,6 g; Acide nicotinique : $3 \mathrm{~g}$; Pantothénate de $\mathbf{C a}: 1,5 \mathrm{~g}$; Vitamine B6:0,2 $\mathrm{g}$; Vitamine B12: $2 \mathrm{mg}$; Vitamine $\mathrm{K} 3: 0,1 \mathrm{~g}$; Acide folique : $0,1 \mathrm{~g}$; Chlorure de choline $50 \%: 100 \mathrm{~g}$. 
La composition et les caractéristiques analytiques des régimes de base sans supplémentation sont présentées dans le tableau 3.

La signification statistique des différentes mesures (consommation, vitesse de croissance et efficacité alimentaire) est testée par analyse de variance, et la comparaison entre les moyennes est réalisée à l'aide du test de Duncan (SNEDECor \& CoCHRAN, 1967).

\section{TABLEAU 3}

Composition et caractéristiques des régimes de base.

Expérience $n^{\circ} 2$.

Composition and characteristics of basal diets.

Experiment 2.

\begin{tabular}{|c|c|c|c|}
\hline Régimes & 1 & 2 & 3 \\
\hline Maïs - Maize & 48,5 & 56,75 & 65 \\
\hline Gluten de maïs 60 - Maize gluten. & 3 & 3 & 3 \\
\hline Lupin - Lupin $\ldots \ldots \ldots \ldots \ldots$ & 35 & 28 & 21 \\
\hline Farine de viande 50 - Meat meal & 6 & 6 & 6 \\
\hline Huile de maïs - Maize oil ...... & 4.5 & 3,25 & 2 \\
\hline Suif - Tallow ............... & 1,5 & 1,5 & 1,5 \\
\hline $\mathrm{NaCl}, \ldots \ldots \ldots \ldots \ldots \ldots \ldots \ldots \ldots \ldots \ldots$ & 0.3 & 0,3 & 0,3 \\
\hline Carbonate de $\mathrm{Ca}-\mathrm{Ca}$ carbonate $\ldots \ldots \ldots \ldots \ldots$ & 0.275 & 0.275 & 0,275 \\
\hline Phosphate bicalcique - Dicalcium phosphate . . & 0.650 & 0,650 & 0,650 \\
\hline Oligo éléments - Trace elements $(*) \ldots \ldots \ldots$ & 0.050 & 0,050 & 0,050 \\
\hline Vitamines - Vitamins $(* *) \ldots \ldots$ & 0,225 & 0,225 & 0,225 \\
\hline Caractéristiques hors supplémentation - Characteristics & & & \\
\hline Energie métabolisable $\mathrm{kgcal} / \mathrm{kg}$ - Metabolizable energy & 3190 & 3190 & 3.190 \\
\hline Protéines brutes - Crude protein $(\mathrm{N} \times 6,25) \ldots \ldots \ldots$ & 20 & 18.5 & 17,1 \\
\hline Lysine $(p .100) \ldots \ldots \ldots \ldots \ldots \ldots \ldots \ldots \ldots \ldots$ & 0,86 & 0.77 & 0,68 \\
\hline Méthionine + Cystine (p. 100) $\ldots \ldots \ldots \ldots \ldots$ & 0,56 & 0,54 & 0,53 \\
\hline Tryptophane - Tryptophan (p. 100) & 0.16 & 0,14 & 0,13 \\
\hline Thréonine (p. 100) ........... & 0.70 & 0,65 & 0,60 \\
\hline
\end{tabular}

(*), (**) Voir tableau 1.

See table 1 .

\section{Résultats}

\section{A. Expérience 1}

Les résultats de la première expérience sont présentés dans le tableau 4.

La consommation alimentaire n'est pas affectée lorsque le régime maïs - tourteau de soja est supplémenté ou non avec 0,5 p. 100 de L-Arginine. De même, l'introduction de 40 p. 100 de lupin doux dans un aliment finition correctement supplémenté en acides aminés essentiels (lysine, méthionine et tryptophane) ne modifie pas la consommation alimentaire par rapport à un régime maïs-tourteau de soja. En revanche, un apport excessif de lysine et de méthionine au régime maïs-lupin diminue les quantités d'aliments ingérées. 


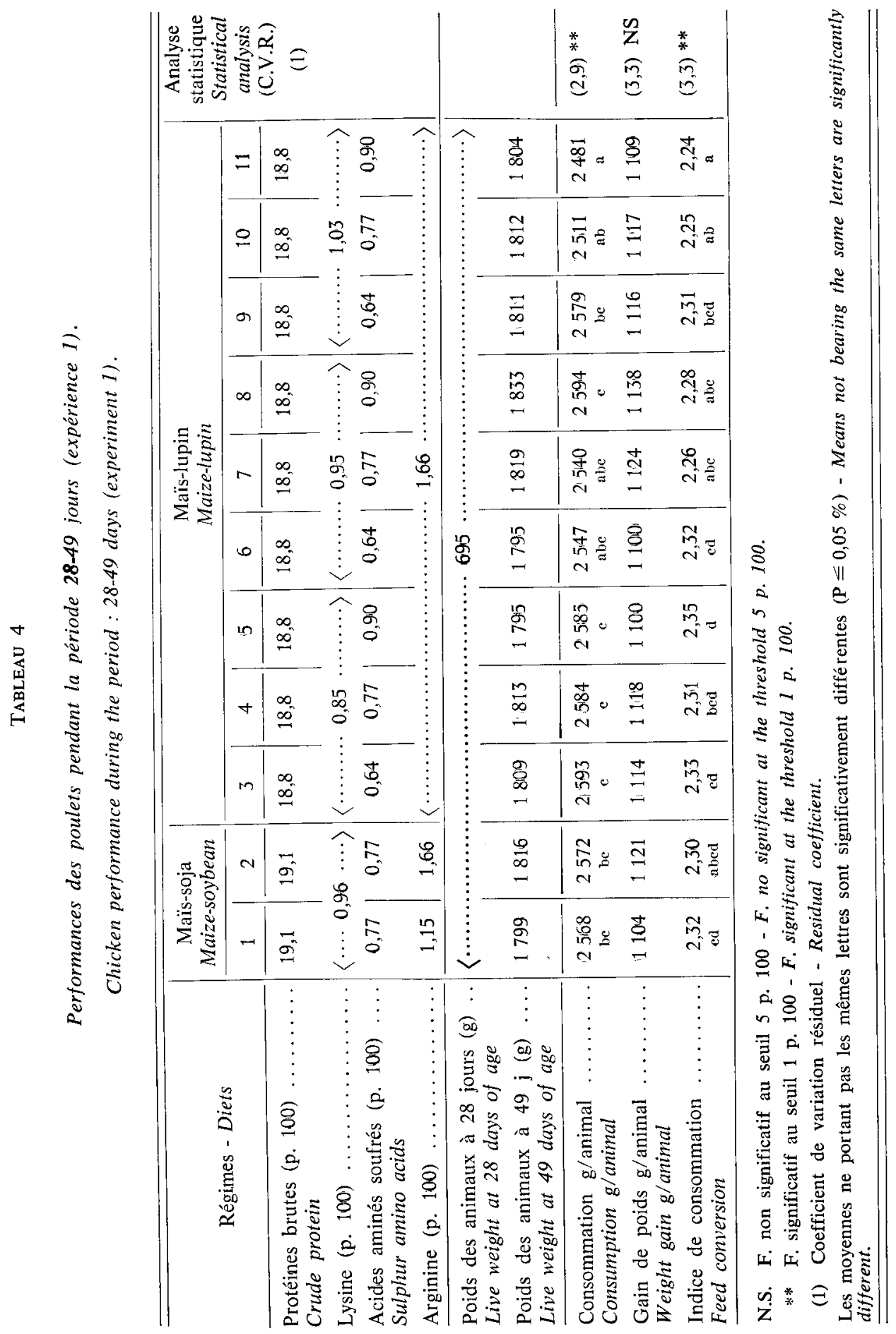


Les vitesses de croissance obtenues dans les lots ne sont pas significativement différentes.

Les indices de consommation varient de 2,35 à 2,24 ; less valeurs les plus faibles sont obtenues avec des aliments maïs-lupin contenant respectivement 0,95 et 0,77 p. 100 de lysine et d'acides aminés soufrés (fig. 1).

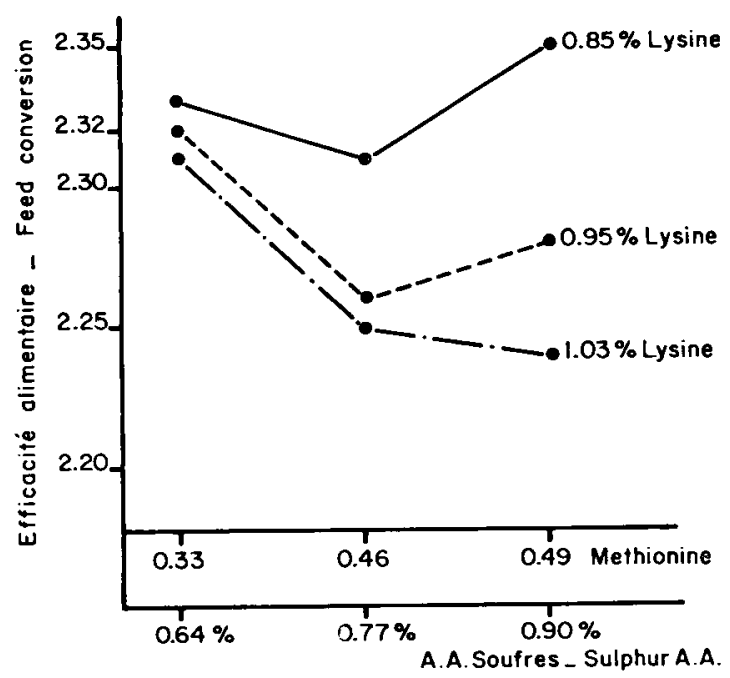

FIG. 1

Efficacité alimentaire du régime mä̈s-lupin

en fonction des apports de lysine et de méthionine (expérience 1).

Efficiency of maize-lupin diet in relation with lysine and methionine levels (experiment l)

\section{B. Expérience 2}

Les résultats de la deuxième expérience font lobjet du tableau 5.

Les gains de poids diffèrent significativement.

La réduction du taux protéique de 18,5 à 17 p. 100, sans supplémentation en acides aminés de synthèse, entraîne une baisse de la vitesse de croissance. Les additions de lysine, méthionine et tryptophane sont dautant plus efficaces que le taux protéique est bas. Ces résultats montrent que, quel que soit le taux protéique, un régime à base de maïs et de lupin apparaît subdéficient en ces trois acides aminés. Dans la mesure où l'équilibre en acides aminés essentiels est réalisé, le taux protidique n'apparaît exercer aucune influence. Dans nos conditions expérimentales, les gains de poids sont identiques dans les lots renfermant $17,18,5$ et 20 p. 100 de protéines lorsqu'ils sont rééquilibrés en lysine, méthionine et tryptophane. 


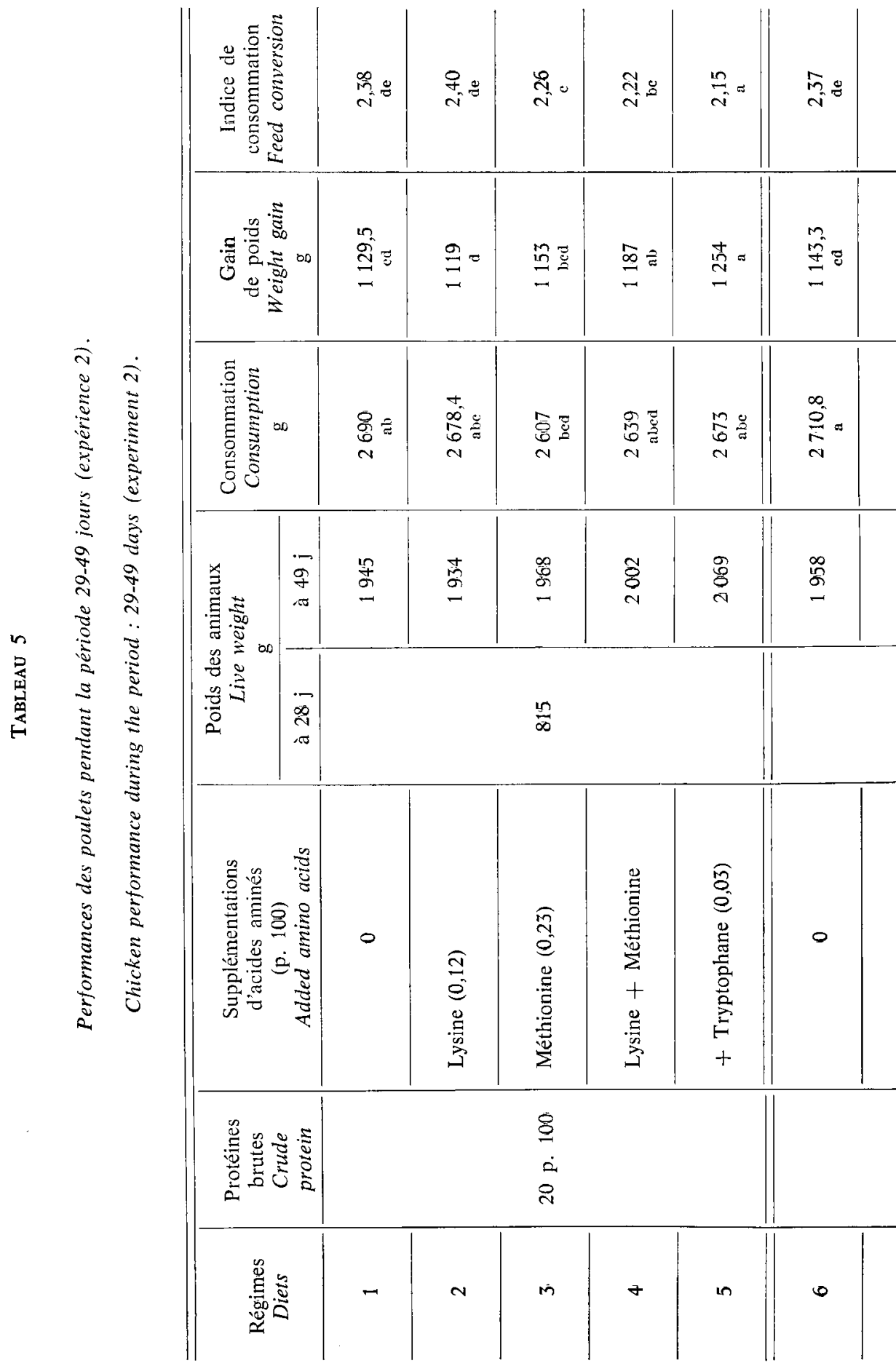


Enfin, les résultats des indices de consommation montrent que les efficacités des régimes entre 17 et 20 p. 100 de protéines sont uniquement liées aux apports des acides aminés essentiels (lysine, méthionine et tryptophane). La supplémentation en thréonine apparaît sans effet.

\section{Discussion et conclusion}

Les résultats de l'expérience 1 montrent que la substitution totale du tourteau de soja par des graines de lupin (L. Albus var. Kalina) dans un régime de finition équilibré en acides aminés n'altère pas les performances des oiseaux. Sur une période expérimentale identique, LaCASSAGNe (1979) avait noté que l'introduction de 40 p. 100 de lupin dans l'aliment diminuait les quantités d'aliments ingérées ainsi que les performances de croissance. L'effet éventuel d'un excès d'arginine, lié à l'introduction du lupin, ne semble pas se vérifier dans notre essai. Dailleurs, l'adjonction de 0,5 p. 100 d'arginine de synthèse dans le régime maïs-tourteau de soja n'a pas entrâ̂né de dégradation des performances. Selon ce même auteur, cette dégradation des performances pourrait aussi être liée à une indisponibilité totale ou partielle de l'acide folique dans le lupin.

Quoi qu'il en soit, la limite de tolérance du lupin doux chez le poulet de chair est grande. Chez l'animal en croissance, l'introduction de lupin jaune doit être modulée en fonction de l'âge selon Vogt, Harnisch \& KRIEG (1979), et varie de 10 p. 100 à une semaine, à 30 p. 100 à quatre semaines. Sur une même période d'étude, le lupin blanc (Kalina) pourrait être incorporé à plus de 30 p. 100 (LACASSAGNE, 1979). D'ailleurs, les travaux de Guillaume, Chenieux \& Rideau (1979) et Muindi \& RundGREN (1981) montrent que l'azote protéique de cette matière première est bien utilisé par les poulets. Le lupin bleu (L. Angustifolius) peut se substituer aux autres sources protéiques sans affecter les performances de croissance à condition d'être utilisé à un taux inférieur à 24 p. 100 dans un régime équilibré en acides aminés (YULE \& MC BRIDE, 1976).

Utilisé comme seule source protéique, en complément de céréales, le lupin exerce un effet dépressif qui est corrigé totalement ou presque par l'addition de méthionine et de lysine (Perez Cuesta \& Perez Herndandez, 1978). Ainsi, l'expérience 1 montre-t-elle que l'efficacité alimentaire du régime est optimale lorsque les niveaux de lysine et d'acides aminés soufrés sont respectivement de 0,95 et 0,77 p. 100 .

Pendant la même période expérimentale, nous avons envisagé de réduire le taux protéique de la ration en diminuant l'introduction du lupin (expérience 2). Entre 20 et 17 p. 100 de protéines, les performances des animaux ne diffèrent pas entre elles lorsque les régimes sont équilibrés en lysine, méthionine et tryptophane. Pour un régime contenant 3200 E.M./ kg il apparaît subcarencé en tryptophane lorsque le taux devient inférieur ou égal à 0,16 p. 100. Selon Hunchar \& Thomas (1976), le besoin en tryptophane du poulet de chair pendant la période de finition serait de 0,054 mégacalorie, soit 0,16 p. 100 pour notre régime considéré.

Dans le contexte économique actuel, la substitution totale du tourteau de soja par du lupin doux pose donc le problème de sa déficience en tryptophane; le prix d'intérêt de cet acide aminé de synthèse est trop élevé pour l'incorporer dans l'aliment. 
Néanmoins, cette légumineuse peut être introduite en quantité importante dans l'aliment, et dans ce cas, les supplémentations de lysine et méthionine sont suffisantes pour optimiser les performances. Ce protéagineux peut aussi être associé à une source riche en tryptophane, tel qu'un concentrat de protéines de luzerne par exemple, afin d'éviter une subdéficience en cet acide aminé (LARBIER \& BLuM, 1981). Cette même association, rééquilibrée en lysine, constitue aussi une solution originale pour la couverture des besoins azotés du porc en croissance (Bourdon, Perez \& CALMES, 1980).

Apparemment, l'apport important de cellulose lié à l'introduction de lupin dans le régime n'est pas préjudiciable aux performances du poulet de chair.

Accepté pour publication en octobre 1982.

\section{Remerciements}

L'auteur tient à remercier Madame N. Marchand, ainsi que Madame J. Salvert et son équipe, pour l'aide technique qu'elles ont apportée dans ce travail.

\section{Summary}

\section{Lupin utilization (Lupinus albus L.) in broilers during finishing period}

The purpose of these two experiments was to study the introduction of lupin (Lupinus albus $\mathbf{L}$.) in finishing diets for broilers (4-7 weeks).

In experiment 1 , all diets provided high levels of protein $(19 \mathrm{p} .100)$. The control diet was a practical type maize-soybean meal, supplemented or not with arginine. Other diets contained lupin $(40 \mathrm{p} .100)$ in the place of soybean meal, and were balanced in essential amino acids with the addition of L-lysine $\mathrm{HCl}$, DL-Methionine and L-Tryptophan. In experiment 2, we studied the effect of a reduction of protein level in maize-lupin-meat meal diets, supplemented or not with synthetic amino acids.

In experiment 1 , the replacement of soybean meal by lupin did not affect the performances, when the diet was balanced in essential amino acids (lysine, methionine and tryptophan) (Table 4). The high level of arginine in relation to lupin introduction in the diet did not reduce the performances. In fact, the addition of arginine to the maize-soybean meal diet leading to an arginin content similar to that of the maize-lupin diet $(1.66 \mathrm{p}$. 100) did not affect performances. In experiment 2 , the reduction of protein from 20 to 17 p. 100 by reducing the level of lupin in the diet led to a decrease in growth performances. However, the simultaneous addition of lysine, methionine and tryptophan allowed to obtain the same performance as with the control diet (Table 5).

\section{Références bibliographiques}

A.E.C., 1978. Document $n^{\circ}$ 4. Energie, acides aminés, vitamines, minéraux. Commentry, France.

Bourdon D., Perez J.M., Calmes R., 1980. Le lupin (Lupinus albus L.) dans l'alimentation du porc : valeur énergétique et azotée et conditions d'utilisation. Journ. Rech. porc. Fr., Paris, I.N.R.A.I.T.P. éd., 245-263. 
Gladstones J.S., 1977. The narrow-leafed lupin in Western Australia. Bulletin 3990, Western Australian Department of agriculture, 3-37.

Guillaume J., Chenieux J.C., Rideau M., 1979. Feeding value of Lupinus albus L. in chicken diets. Nutr. Rep. Int., 20, 57-65.

Hunchar G.J., Thomas O.P., 1976. The tryptophan requirement of male and female broilers during the 4-7 week period. Poult. Sci., 55, 379-383.

LACASSAGNe L., 1979. Valeur alimentaire du lupin blanc doux, variété Kalina, chez le poulet en croissance. In : Matières premières et alimentation des volailles, I.N.R.A., Station de Recherches avicoles, Séances de travail des 18 et 19 octobre 1979, 43-56.

Larbier M., Blum J.C., 1981. Remplacement du tourteau de soja par de la farine de viande et des associations de protéagineux dans l'alimentation du poulet de chair. Ann. Zootech., 30, 335-346.

Muindi P.J., Rundgren M., 1981. Investigations on the protein quality and alkaloid content of Lupinus albus L. cv Kalina grown in Tanzania. Nutr. Rep. Int., 23, 391-399.

Perez Cuesta M., Perez Hernandez M., 1978. Rendimientos compartivos en pollos para carne de la harina de semillas de $L$. albus Neuland y de la torta de sajo. Proc. $16^{e}$ Congrès Mondial d'Aviculture, Rio de Janeiro, 545-551.

SNedecor G.W., Cochran W.G., 1967. Statistical methods (6 $6^{\circ}$ édition). Iowa State University Press, Ames, Iowa, 302-307.

Vogt H., Harnisch S., Krieg R., 1979 a. Der Einsatz von Süsslupinenschrot im Geflügelfutter. Arch. Geflïgelk., 43, 229-238.

Vogt H., Harnisch S., Krieg R., 1979 b. Der Einsatz von Erbsenschrot im Geflügelfutter. Arch. Geflïgelk., 43, 195-199.

YUlE W.J., MC BRIDE R.L., 1976. Lupin and rapeseed meals in poultry diets : effect on broiler performance and sensory evaluation of carcasses. Br. Poult. Sci., 17, 231-239. 